\title{
Efficacy and Safety of Elbasvir/Grazoprevir Combination Therapy for Chronic Hepatitis C
}

\author{
Satoru Hagiwara and Masatoshi Kudo* \\ Department of Gastroenterology and Hepatology, Japan
}

Received: 制 January 03, 2019; Published: 制January 22, 2019

*Corresponding author: Masatoshi Kudo, Department of Gastroenterology and Hepatology, Japan

\begin{abstract}
Objective: A phase III clinical study of combination therapy with elbasvir (EBR) and grazoprevir (GZR) was conducted in Japan, and the additive therapeutic effects were obtained. In this study, we report safety and efficacy of EBR/GZR combination therapy in a real-world practice.
\end{abstract}

Methods: Sixty-two patients with chronic hepatitis C received EBR/GZR combination therapy from November 2016. Patients who achieved sustained virologic responses at 12 weeks (SVR12) were included in the study. A total of 40 patients were enrolled in this retrospective study. Thirtyeight patients were naive for direct-acting antiviral (DAA) therapy and two patients were previously treated with DAA.

Results: All of 38 DAA-naïve patients achieved SVR12 by EBR/GZR combination therapy, whereas two patients with DAA failure prior to this treatment did not achieve SVR12. A total of 21 patients (52.5\%) had cardiovascular diseases, and 5 patients (12.5\%) had advanced kidney diseases with eGFR $<30$. All of these patients achieved SVR12, and the treatment was performed safely even in the presence of cardiovascular and kidney diseases.

Conclusion: Clinical efficacy and safety of EBR/GZR combination therapy have been confirmed in this study. EBR/GZR combination therapy is extremely effective in chronic hepatitis C patients naive for DAA treatments. Administration of EBR and GZR therapy is safe in patients bearing cardiovascular and kidney disease.

Abbreviations: DAAs: Direct-acting antivirals; EBR: Elbasvir; GZR: Grazoprevir

\section{Introduction}

Eradication of hepatitis $\mathrm{C}$ virus is crucial for risk reduction of human hepatocarcinogenesis [1-3]. Direct-acting antiviral (DAA) agents such as telaprevir (TVR) [4-6] and simeprevir (SMV) [711] are the first ones that were introduced for the treatment of patients with chronic hepatitis $\mathrm{C}$ virus (HCV) infection from 2011 to 2013. Although these DAAs have improved the overall efficacy of chronic hepatitis C (CHC) treatment, TVR or SMV was used in combination with pegylated interferon (PEG-IFN) and ribavirin (RBV). A significant population of patients with CHC were compelled to discontinue or skip scheduled treatments with triple therapy consisting of PEG-IFN, RBV, and TVR or SMV since this treatment regimen caused a wide variety of side effects such as anemia and liver dysfunction. Moreover, administration of PEGIFN is accompanied by the appearance of high fever and general malaise, which sometimes impair the patients' quality of life. To overcome such disadvantages of triple therapy consisting of PEGIFN, RBV, and TVR or SMV, combination therapy with asunaprevir
(ASV) and daclatasvir (DCV) was approved in 2014 as the first IFN-free regimen [12-15]. Compared with treatment regimens including IFN, this combination therapy can be applied to a broad range of patients.

Even the elderly patients with comorbidities have become good candidates for this IFN-free regimen. Different from IFN-based regimens, the efficacy of the combination therapy with ASV and DCV was not affected by patient characteristics, such as sex and age, or the presence of liver fibrosis and IL28B polymorphism. Although the combination therapy with ASV and DCV was safe with minimal adverse effects, the efficacy of this regimen was dependent upon the presence of baseline viral mutations that conferred resistance to the treatment. In addition, some patients were unable to continue the treatment due to side effects such as liver dysfunction. Combination therapy with sofosbuvir (SOF) and ledipasvir (LDV) is a second generation IFN-free regimen that can eliminate HCV bearing mutations resistant to ASV and DCV. A phase 
III trial of combination therapy with SOF and LDV in Japan resulted in a remarkable success in the achievement of sustained viral responses (SVR) due to an additive effect of the two drugs [16]. Despite of extremely high elimination rates in CHC patients treated with SOF and LDV, two major concerns regarding this combination have been raised. Firstly, SOF is excreted by the kidneys and thus its use was contraindicated in patients with advanced kidney diseases. Secondly, combination therapy with SOF and LDV may have some toxic effects on cardiac functions leading to QT interval prolongation [17]. Combination therapy with elbasvir (EBR) and grazoprevir (GZR) is a third generation IFN-free regimen for CHC. A phase III trial of combination therapy with EBR and GZR has provided evidence that, this combination regimen is safe even in patients with advanced kidney diseases [18]. In the present study, we examined the efficacy and safety of EBR/GZR combination therapy in patients with CHC. Our results suggest that combination therapy with EBR and GZR is very effective even in elderly patients bearing impaired heart and kidney functions.

\section{Patients and Methods}

\section{Patients and Treatment Schedule}

Sixty-two patients with $\mathrm{CHC}$ received combination therapy with EBR and GZR at Kindai University Hospital from November 2016. Patients were included in this retrospective study if their sustained virologic responses at 12 weeks (SVR12) were able to be evaluated. A total of 40 patients were enrolled in the study. The combination therapy was administered for a total of 12 weeks, followed by 12 weeks observation. Patients received oral administration of 50 $\mathrm{mg}$ of EBR and $100 \mathrm{mg}$ of GZR once daily for a total of 12 weeks. Ethical permission of this study was granted by the Review Boards of Kindai University Faculty of Medicine.

\section{Quantification of HCV-RNA}

HCV-RNA levels were measured using the Roche COBAS Taqman test with lower and upper limits of quantitation of $15 \mathrm{IU} / \mathrm{mL}$ and $8.0 \log \mathrm{IU} / \mathrm{mL}$.

\section{HCV Mutation Analysis}

HCV NS5A and NS3/4A mutations that confer drug resistance were examined using PCR-invader, CycleavePCR, and direct sequencing. Serum samples isolated from 13 patients were subjected to HCV mutation analysis.

\section{Results}

\section{Patients}

Sixty-two patients with CHC received combination therapy with EBR and GZR at our hospital from November 2016. Forty patients were included in the study, as their SVR12 were evaluable. As shown in Table 1 the median age of the patients was 74 years; $29(73 \%)$ were over the age of 65 and 18 (45\%) were over the age of 75 . Thus, the number of elderly patients in our study was higher than that in the phase III clinical trial conducted in Japan [18]. In addition, 12 patients (30\%) had cirrhosis and 7 (18\%) had a previous history of liver cancer that was cured. There were 2 patients (5\%) who had been previously treated with DAA; one patient was refractory to combination therapy with DCV and ASV, whereas the other patient had experienced breakthrough with DCV and ASV and then relapsed with subsequent SOF and LDV (Table 1)

\section{Baseline Resistance-Conferring Mutations in NS5A}

The presence of NS5A mutations was examined in 13 patients. NS5A mutations were not detected in six patients (15\%), seven patients (18\%) had Y93 mutations, and two patients (5\%) had L31 mutations (Table 1).

Table 1: Patient background.

\begin{tabular}{|c|c|}
\hline \multicolumn{2}{|c|}{ Patient background } \\
\hline Age, years & $\mathbf{n = 4 0}$ \\
\hline Median (range) & $74(19-86)$ \\
\hline$>65$ years, $\mathrm{n}(\%)$ & $29(73 \%)$ \\
\hline$>75$ years, $\mathrm{n}(\%)$ & $18(45 \%)$ \\
\hline Male sex, $\mathrm{n}(\%)$ & $18(45 \%)$ \\
\hline Cirrhosis, $\mathrm{n}(\%)$ & $12(30 \%)$ \\
\hline History of HCC, $\mathrm{n}(\%)$ & $7(18 \%)$ \\
\hline Previous DAAs therapy, n (\%) & $2(5 \%)$ DCV/ASV;1, DCV/ \\
& ASV $\rightarrow$ SOF $/ \mathrm{LDV} ; 1$ \\
\hline NS5A mutation & \\
\hline Wild, $\mathrm{n}(\%)$ & $6(15 \%)$ \\
\hline Y93, $\mathrm{n}(\%)$ & $7(18 \%)$ \\
\hline L31, $\mathrm{n}(\%)$ & $2(5 \%)$ \\
\hline N.E., $\mathrm{n}(\%)$ & $27(68 \%)$ \\
\hline
\end{tabular}

\section{Virologic Response}

All 40 patients (100\%) achieved end-of-treatment-response (ETR). Two patients had relapsed during the subsequent 12-week observation period. Thus, SVR12 was achieved in 38 patients (95\%) (Figure 1).

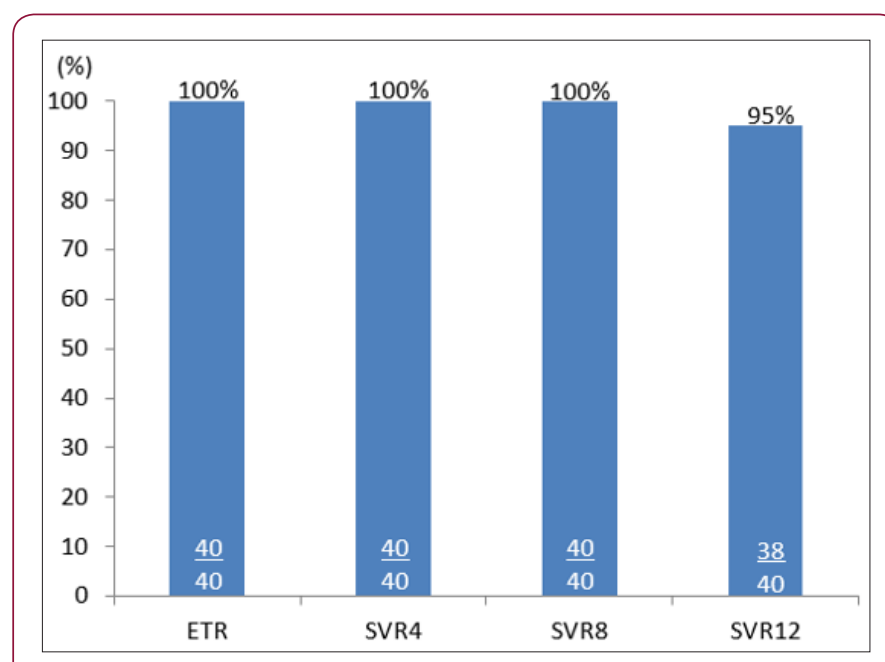

Figure 1: SVR12 was achieved in 38 patients (95\%).

\section{Virologic Relapse}

Two patients who had previously been treated with DAA relapsed after the 12-week treatment. One of the patients was refractory to combination treatment with DCV and ASV and had acquired Y93H $(100 \%)$ and $\mathrm{L} 31 \mathrm{M}, \mathrm{V}(>20 \%)$ mutations prior to the administration 
of EBR and GZR. The other patient achieved breakthrough with DCV and ASV and then relapsed with subsequent combination treatment with SOF and LDV. This patient also had resistance mutations of Y93H (>20\%) and L31M, V (>20\%) prior to the administration of

Table 2: Characteristics of patients who did not achieve SVR.
EBR and GZR (Table 2). Thus, the presence of baseline mutations in NS5A resistant to DAA was strongly associated with relapse after EBR and GZR in patients with DAA failure.

\begin{tabular}{|c|c|c|c|c|c|c|c|}
\hline Age & Sex & $\begin{array}{l}\text { Previous DAA } \\
\text { therapy }\end{array}$ & Cirrhosis & $\begin{array}{c}\text { History of HCC } \\
\text { treatment }\end{array}$ & IL28B Rs 8099917 & $\begin{array}{l}\text { Pre-treatment } \\
\text { resistance }\end{array}$ & Side effects \\
\hline 73 & Female & Refractory (DCV/ASV) & No & No & TT & $\begin{array}{c}\text { Y93H }(100 \%) \mathrm{L} 31 \mathrm{M}, \mathrm{V} \\
(>20 \%)\end{array}$ & None \\
\hline 79 & Female & $\begin{array}{c}\text { BT (DCV/ASV) } \\
\rightarrow \\
\text { Relapse }(\text { SOF/LDV) }\end{array}$ & No & No & TG & $\begin{array}{c}\text { Y93H (>20\%)L31V } \\
(>20 \%)\end{array}$ & None \\
\hline
\end{tabular}

Baseline NS5A (Y93) Resistance-Conferring Mutation and Antiviral Efficacy of EBR and GZR Therapy in DAAnaïve patients

Five DAA-naïve patients had Y93H mutations in NS5A. Four patients had a high frequency of resistance-conferring mutations $(\geq 50 \%)$. However, all patients achieved SVR12 (Table 3). Thus, the presence of baseline mutations in NS5A resistant to DAA did not affect the rates of SVR12 in DAA-naive patients treated with EBR and GZR.

Table 3: Baseline resistance-conferring mutations in NS5A in DAA-naïve patients.

\begin{tabular}{|c|c|c|c|c|c|c|c|}
\hline Age & Sex & $\begin{array}{c}\text { Previous DAA } \\
\text { therapy }\end{array}$ & L31 & Y93H & Cirrhosis & eGFR & SVR \\
\hline 77 & Male & None & $(-)$ & Y93H (50\%) & Yes & 7 & SVR12 \\
\hline 82 & Female & None & $(-)$ & Y93H (90\%) & Yes & 58 & SVR12 \\
\hline 70 & Male & None & $(-)$ & Y93H (100\%) & No & 5 & SVR12 \\
\hline 42 & Male & None & $(-)$ & Y93H (50\%) & No & 61 & SVR12 \\
\hline 78 & Female & None & $(-)$ & Y93H (1-20\%) & No & 65 & SVR12 \\
\hline
\end{tabular}

Anti-viral Efficacy and Safety of EBR/GZR Therapy for Patients with Cardiovascular Disease

A total of 21 (52.5\%) among the 40 patients had cardiovascular diseases. All 21 patients achieved SVR12, and none had side effects, including heart disease (Figure 2). Thus, combination therapy with EBR and GZR is safe even patients with cardiovascular diseases.

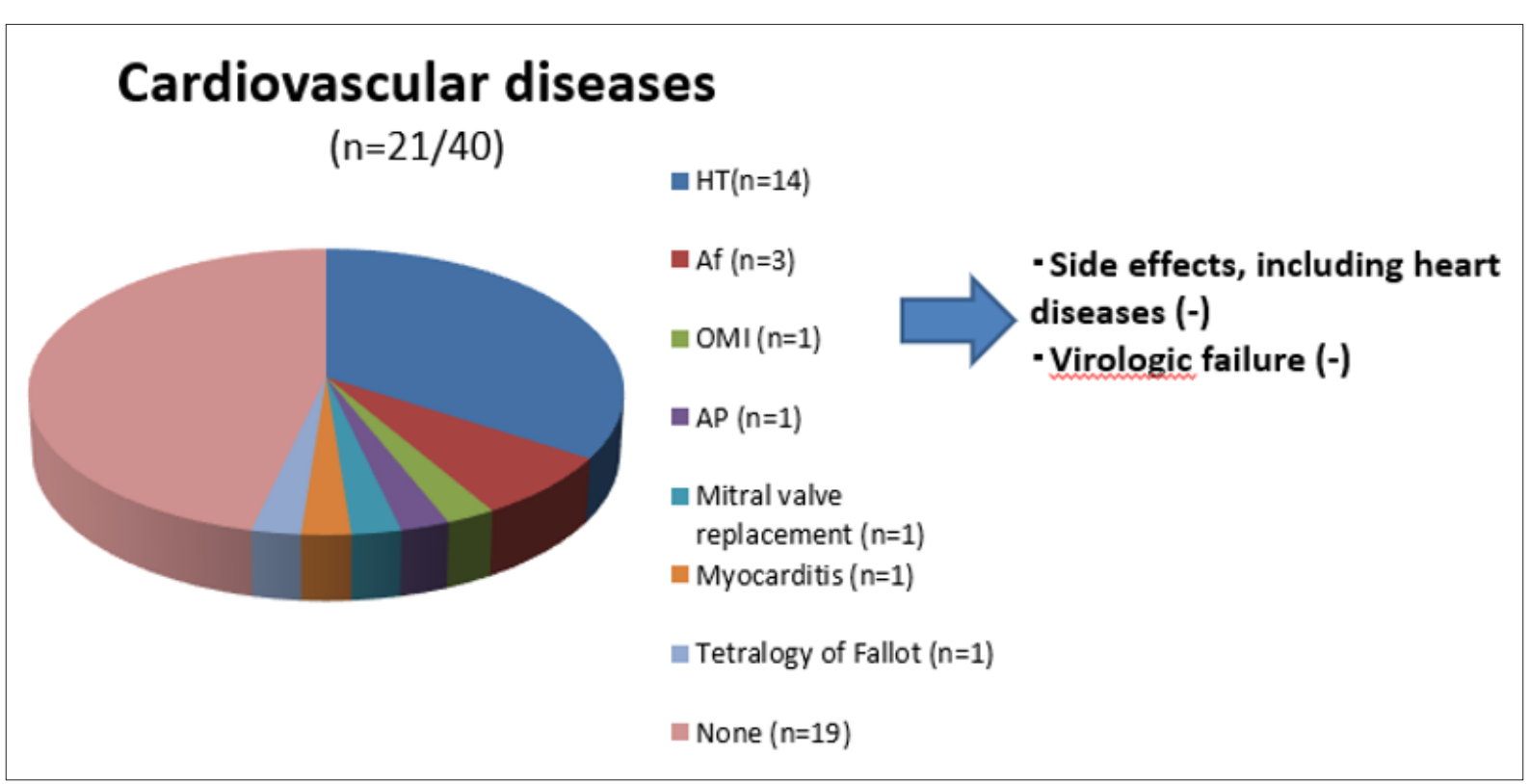

Figure 2: All 21 patients achieved SVR12, and none had side effects, including heart disease. 
Anti-viral Efficacy and safety of EBR/GZR Therapy for Patients with Advanced kidney diseases (eGFR<30)

Table 4: Side effects leading to discontinuation of the therapy.

\section{N, (\%)}

1 (2.5\%), increased ALT level

Five of the 40 patients had advanced kidney diseases with eGFR<30. All 5 patients achieved SVR12, and none had side effects (Table 4). Thus, combination therapy with EBR and GZR is safe even patients with advanced kidney diseases.

\section{Side Effects leading to Discontinuation of EBR/GZR Therapy}

Combination therapy with EBR and GZR was discontinued in one patient $(2.5 \%)$ due to an increased level of alanine aminotransferase (ALT, $261 \mathrm{IU} / \mathrm{mL}$ ) at week 10. The treatment was re-initiated after the reduction of ALT levels to complete the 12week regimen (Table 5).

Table 5: Patients with advanced kidney diseases (eGFR<30).

\begin{tabular}{|c|c|c|c|c|c|c|c|c|}
\hline Age & Sex & Cirrhosis & $\begin{array}{c}\text { Baseline } \\
\text { mutation }\end{array}$ & SVR & HD & eGFR (pre) & eGFR (post) & Side effects \\
\hline 79 & Male & No & N.E. & SVR12 & No & 21 & 20 & None \\
\hline 77 & Male & Yes & $\begin{array}{c}\text { Y93H (50\%) } \\
\text { L31(-) }\end{array}$ & SVR12 & Yes & 7 & None \\
\hline 70 & Male & No & $\begin{array}{c}\text { Y93H (100\%) } \\
\text { L31(-) }\end{array}$ & SVR12 & Yes & 5 & 6 & None \\
\hline 55 & Male & No & N.E. & SVR12 & Yes & 6 & 4 & None \\
\hline 74 & Male & Yes & N.E. & SVR12 & Yes & 8 & 9 & None \\
\hline
\end{tabular}

\section{Discussion}

The Japanese phase III trial of combination treatment with EBR and GZR in DAA-naïve patients revealed that $96.5 \%$ of HCVinfected patients with liver cirrhosis and $97.1 \%$ of patients with compensated liver cirrhosis achieved SVR12 [18]. As for the presence of baseline NS5A mutations conferring resistance to DAA, the Japanese phase II and phase III trials disclosed that $93.1 \%$ of patients bearing such NS5A mutations achieved SVR12, suggesting that the presence of mutations do not affect the elimination rates of HCV in this combination therapy. [18]. In line with these trials, 38 of 40 patients (95\%) achieved SVR12 in this study. The two patients who did not achieve SVR12 experienced treatment failure in prior DAA treatment. Moreover, they acquired mutations in Y93 and L31 prior to the initiation of EBR and GZR therapy. Thus, DAA-experienced patients who have already acquired resistance due to multiple mutations in NS5A may not be suitable candidates for EBR and GZR therapy. On the other hand, all of 38 DAA-naïve patients achieved SVR12 in this study. Although five of these patients had $\mathrm{Y} 93 \mathrm{H}$ mutations and 4 had a high frequency ( $\geq 50 \%$ ) of resistance-conferring mutations, they all achieved SVR12. This suggests that the presence of resistance-conferring mutations does not influence the efficacy of EBR and GZR therapy in DAAnaive patients. Although our study included a higher proportion of elderly patients and those with more advanced liver fibrosis as compared with the Japanese phase III trial, all of our patients achieved SVR. Therefore, combination therapy with EBR and GZR may be an effective treatment option for DAA-naïve patients with advanced liver cirrhosis. This study population consisted of a high proportion of patients with cardiovascular diseases.

It should be emphasized that combination therapy with EBR and GZR led to achievement of SVR 12 in all patients with cardiovascular diseases and none of such patients discontinued this treatment due to cardiovascular side effects. These data are in stark contrast with those of previous studies regarding combination therapy with SOF and LDV in which such treament may have some toxic effects on cardiac functions leading to QT interval prolongation [17]. Furthermore, several reports suggest that HCV infection and cirrhosis may cause QT interval prolongation and cardiovascular diseases such as angina [19-20]. Indeed, a significant population of patients who received combination therapy with SOF and LDV developed cardiovascular side effects, leading to discontinuation of the treatment. Our findings strongly suggest that combination therapy with EBR and GZR is safe and effective treatment option for patients with heart diseases. Both EBR and GZR are metabolized by CYP3A and can be administered safely to patients with kidney diseases. In phase II and phase III trials (C-SURFER), combination therapy with EBR and GZR treatment led to SVR12 in $99.1 \%$ of the patients with advanced stages of chronic kidney disease without serious adverse events [21].

In the present study, 5 of the 40 patients had advanced kidney diseases with eGFR<30, and all 5 patients achieved SVR12 without developing any side effects (Table 4). Thus, consistent with previous reports, this combination therapy was found to be a safe and effective treatment option for patients with advanced kidney diseases. These dat altogether strongly support the idea that combination therapy with EBR and GZR is safe and effective even in elderly $\mathrm{CHC}$ patients with cardiovascular and kidney diseases. One patient $(2.5 \%)$ developed side effects that led to discontinuation of the therapy due to the elevation of serum ALT levels. The patient skipped the treatment for 2 weeks and then was re-started after the confirmation of reduction of ALT levels to complete the 12week regimen (Table 5). Clinical course of this patient suggest that close and careful monitoring of liver enzymes is might be required in combination therapy with EBR and GZR. In conclusion, we demonstrated that combination therapy with EBR and GZR is an effective treatment option for DAA-naïve patients bearing 
advanced kidney diseases and cardiovascular diseases. Given the fact that an SVR rate of over $95 \%$ was achieved with DAAs further studies focusing on improvement of the safety are required. It is no doubt that combination therapy with EBR and GZR is one of such candidates that can be easily applied to the elderly CHC patients bearing a wide variety of comorbidities.

\section{References}

1. Owusu Sekyere S, Schlevogt B, Mettke F, Kabbani M, Deterding K, et al. (2018) HCC immune surveillance and antiviral therapy of hepatitis C virus infection. Liver Cancer p. 7.

2. Koda M, Tanaka S, Takemura S, Shinkawa H, Kinoshita M, et al. (2018) Long-term prognostic factors after hepatic resection for hepatitis $C$ virus-related hepatocellular carcinoma, with a special reference to viral status. Liver Cancer 7(3): 261-276.

3. Finkelmeier F, Dultz G, Peiffer KH, Kronenberger B, Krauss F (2018) Risk of de novo hepatocellular carcinoma after HCV treatment with directacting antivirals. Liver Cancer 7(2): 190-204.

4. Kumada H, Toyota J, Okanoue T, Chayama K, Tsubouchi H, et al. (2012) Telaprevir with peginterferon and ribavirin for treatment-naive patients chronically infected with HCV of genotype 1 in Japan. J Hepatol 56(1): 78-84.

5. Jacobson IM, Mc Hutchison JG, Dusheiko G, Adrian M Di Bisceglie, et al. (2011) Telaprevir for previously untreated chronic hepatitis $C$ virus infection. N Engl J Med 364: 2405-2416.

6. Zeuzem S, Andreone P, Pol S, Diago M, Roberts S, et al. (2011) Telaprevir for retreatment of HCV infection. N Engl J Med 364(25): 2417-2428.

7. Hayashi N, Izumi N, Kumada H, Okanoue T, Tsubouchi H, et al. (2014) Simeprevir with peginterferon/ribavirin for treatment-naïve hepatitis C genotype 1 patients in Japan: CONCERTO-1, a phase III trial. J Hepatol 61(12): 219-227

8. Izumi N, Hayashi N, Kumada H, Okanoue T, Tsubouchi H, et al. (2014) Once-daily simeprevir with peginterferon and ribavirin for treatmentexperienced HCV genotype 1 -infected patients in Japan: the CONCERTO-2 and CONCERTO-3 studies. J Gastroenterol 49(5): 941-953.

9. Kumada H, Hayashi N, Izumi N, Okanoue T, Tsubouchi H, et al. (2015) Simeprevir (TMC435) once daily with peginterferon- $\alpha-2 b$ and ribavirin in patients with genotype 1 hepatitis $C$ virus infection: The CONCERTO-4 study. Hepatol Res 45(5): 501-513.

10. Jacobson IM, Dore GJ, Foster GR, Fried MW, Radu M, et al. (2014) Simeprevir with pegylated interferon alfa 2a plus ribavirin in treatmentnaive patients with chronic hepatitis $\mathrm{C}$ virus genotype 1 infection
(QUEST-1): a phase 3, randomised, double-blind, placebo-controlled trial. Lancet 384(9941): 403-413.

11. Manns M, Marcellin P, Poordad F, De Araujo ES, Buti M, et al. (2014) Simeprevir with pegylated interferon alfa $2 \mathrm{a}$ or $2 \mathrm{~b}$ plus ribavirin in treatment-naive patients with chronic hepatitis $\mathrm{C}$ virus genotype 1 infection (QUEST-2): a randomised, double-blind, placebo-controlled phase 3 trial. Lancet 384(9941): 414-426.

12. Chayama K, Takahashi S, Toyota J, Karino Y, Ikeda K, et al. (2012) Dual therapy with the nonstructural protein $5 \mathrm{~A}$ inhibitor, daclatasvir, and the nonstructural protein 3 protease inhibitor, asunaprevir, in hepatitis $C$ virus genotype $1 \mathrm{~b}$-infected null responders. Hepatology 55(3): 742-748.

13. Karino Y, Toyota J, Ikeda K, Suzuki F, Chayama K, et al. (2013) Characterization of virologic escape in hepatitis C virus genotype $1 \mathrm{~b}$ patients treated with the direct-acting antivirals daclatasvir and asunaprevir.J Hepatol 58(4): 646-654.

14. Suzuki Y, Ikeda K, Suzuki F, Toyota J, Karino Y, et al. (2013) Dual oral therapy with daclatasvir and asunaprevir for patients with HCV genotype $1 \mathrm{~b}$ infection and limited treatment options. J Hepatol 58(4): 655-662.

15. Kumada H, Suzuki Y, Ikeda K, Toyota J, Karino Y, et al. (2014) Daclatasvir plus asunaprevir for chronic HCV genotype $1 \mathrm{~b}$ infection. Hepatology 59(6): 2083-2091.

16. Mizokami M, Yokosuka O, Takehara T, Sakamoto N, Korenaga M, et al. (2015) Ledipasvir and sofosbuvir fixed-dose combination with and without ribavirin for 12 weeks in treatment-naive and previously treated Japanese patients with genotype 1 hepatitis C: an open-label, randomised, phase 3 trial. Lancet Infect Dis 15(6): 645-653.

17. Hagiwara S, Nishida N, Watanabe T, Sakurai T, Ida H, et al. (2017) Outcome of Combination Therapy with Sofosbuvir and Ledipasvir for Chronic Type C Liver Disease. Oncology 92(1): 3-9.

18. Kumada H, Suzuki Y, Karino Y, Chayama K, Kawada N (2017) The combination of elbasvir and grazoprevir for the treatment of chronic HCV infection in Japanese patients: a randomized phase II/III study. J Gastroenterol 52(4): 520-533.

19. Gill K, Ghazinian H, Manch R, Gish R (2016) Hepatitis C virus as a systemic disease: reaching beyond the liver. Hepatol Int 10(3): 415-423.

20. Negro F (2014) Facts and fictions of HCV and comorbidities: steatosis, diabetes mellitus, and cardiovascular diseases. J Hepatol 61(1): S69-78.

21. Roth D, Nelson DR, Bruchfeld A, Liapakis A, Silva M, et al. (2015) Grazoprevir plus elbasvir in treatment-naive and treatment-experienced patients with hepatitis C virus genotype 1 infection and stage 4-5 chronic kidney disease (the C-SURFER study): a combination phase 3 study. Lancet 386(10003): 1537-1545.
ISSN: 2574-1241

DOI: 10.26717/BJSTR.2019.13.002403

Masatoshi Kudo. Biomed J Sci \& Tech Res

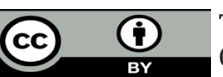

This work is licensed under Creative Commons Attribution 4.0 License

Submission Link: https://biomedres.us/submit-manuscript.php

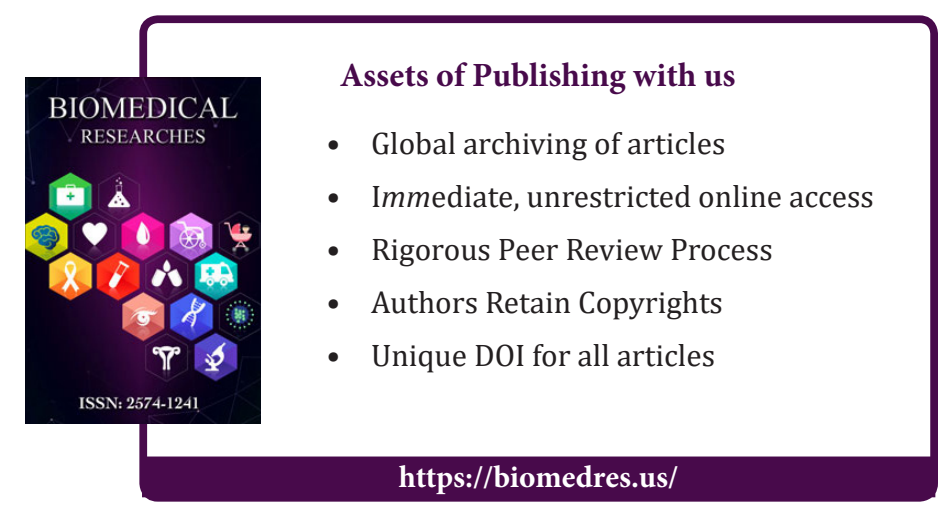

